\title{
The Problematic Lag between FDA Approval of Medical Devices and CMS Coverage
}

\author{
Bradley Knight ${ }^{1}$, Thomas Deering ${ }^{2}$, Michael Gold ${ }^{3}$, Suneet Mittal ${ }^{4}$, and Jagmeet Singh ${ }^{5}$ \\ ${ }^{1}$ Northwestern Medical Center \\ ${ }^{2}$ Piedmont Heart Institute Arrhythmia Center \\ ${ }^{3} \mathrm{MUSC}$ \\ ${ }^{4}$ Valley Health System \\ ${ }^{5}$ Harvard Medical School
}

April 21, 2021

\begin{abstract} and CMS to implement the MCIT rule without delay to ensure timely access to breakthrough technologies

Bradley P. Knight, MD*, Thomas F. Deering, MD**, Michael R. Gold***,

Suneet Mittal, MD****, Jagmeet P. Singh, MD, DPhil ${ }^{* * * * *}$

* Northwestern University, Chicago, IL, bknight@nm.org

** Piedmont Heart Institute, Atlanta GA, Thomas.Deering@piedmont.org

*** Medical University of South Carolina, Charleston, SC, goldmr@musc.edu

****Valley Health, NY, NY, MITTSU@Valleyhealth.com

***** Harvard University, Boston, MA, JSINGH@mgh.harvard.edu
\end{abstract}

Significant changes or cancellation of MCIT could result in limiting access to breakthrough medical technologies that could improve the health and well-being of Medicare beneficiaries. For these reasons, we encourage federal agencies to work together

The Problematic Lag between FDA Approval of Medical Devices and CMS Coverage

Corresponding Author:

Bradley P. Knight, MD

Northwestern Medical Center - Cardiology

251 East Huron Feinberg 8-503E , Chicago, Illinois 60611

bknight@nm.org

Word Count: 939

Dear Readers,

Innovations in medical devices therapy have dramatically improved the care of patients with cardiovascular disease. As cardiologists, we can now offer patients many therapies that improve their quality of life and help them live longer. Modern device therapies are available for our patients as a direct result of the 
ingenuity, contributions, investments, and sacrifices of many groups of people including subjects who have volunteered to participate in clinical trials, health care workers who have enrolled patients, trial sponsors, and federal agencies. In the past, when trials demonstrated safety and efficacy, approval by the Federal Drug Administration (FDA) was predictable as was coverage by the Centers for Medicare and Medicaid (CMS). Most commercial payors followed suit with coverage soon thereafter.

Cardiac electrophysiologists rely heavily on medical devices such as ablation technologies and Cardiac Implantable Electrical Devices (CIEDs) to improve the lives of patients with heart rhythm disorders. Unfortunately, there is an increasing lag between FDA approval of medical devices and CMS Coverage. Too often, novel device-based treatments for cardiovascular diseases, which have been approved by FDA after demonstrating safety and effectiveness, have not been made available to our seniors due to the lack of Medicare coverage. It is rare that a patient can afford the hospital costs associated with our interventions without coverage by CMS or their insurance. Unfortunately, we have seen directly the impact that delays in CMS coverage for heart rhythm therapies have had on our ability to provide the best possible care for patients. One example is the delay between FDA approval of the subcutaneous implantable defibrillator (S-ICD) and coverage by CMS. This was followed by further delays in coverage by private payors. After FDA approval of the S-ICD in 2012, some insurance companies still deny coverage for the S-ICD, except in very specific circumstances $^{1}$. Other examples of medical devices that were not covered by CMS for some time after FDA approval include the Watchman left atrial appendage occlusion device and the Micra leadless pacemaker.

The most common argument made by CMS when it does not provide coverage for a medical device in spite of FDA approval is that CMS believes it must determine that the new therapy is reasonable and necessary for patients over the age of 65 years. This argument is unreasonable in most cases, because Medicare recipients commonly account for a majority of patients enrolled in cardiac device trials. For instance, the average age of subjects enrolled in the recent Micra leadless pacemaker pivotal trial was 76 years. ${ }^{2}$ Other reasons countering the CMS stance are that cardiac disease is common in the elderly, CMS coverage of research devices tested in clinical trials is usually established prior to trial initiation, and physicians at many hospitals are able to enroll only patients in clinical trials who are over 65 year old because commercial carriers usually do not cover the costs associated with participation in clinical trials.

To address this lag between the FDA approval and CMS coverage, several new programs were developed by the United States government over multiple administrations and congresses as a true bipartisan effort. The first program was part of the CURES Act in 2016, which was designed to accelerate access to breakthrough devices that address severely morbid or deadly diseases, with no alternative treatment options. The CMS Administration, after reviewing 362 public comments received during the public comment period, finalized the Medicare Coverage of Innovative Technology (MCIT) rule with an effective date of March 15, $2021^{3}$. This was to ensure four years of Medicare coverage for seniors for these breakthrough devices.

In early March 2021, however, after some concerns were voiced about this program ${ }^{4-5}$, CMS delayed the implementation of the MCIT rule for 60 days and opened a new public comment period that ends on April 16,2021 , questioning the validity of the program and the rule making process. One concern expressed by CMS is the volume of devices designated as breakthrough, which is over 400 . While we fully support an approval process that ensures that a reasonable degree of efficacy for breakthrough technologies has been demonstrated before approval, it is important to note that fewer than $10 \%$ of these devices have been approved at this time, only one quarter of the devices are for the treatment of cardiovascular disease, many are for highly select populations, and many of these may never be approved. Instead, we should be celebrating the increasing volume of new innovative technologies to provide new important options to treat these diseases while often reducing the cost.

Significant changes or cancelation of MCIT could result in limiting access to breakthrough medical technologies that could improve the health and well-being of Medicare beneficiaries. For these reasons, we encourage federal agencies to work together and CMS to implement the MCIT rule without delay to ensure timely access to breakthrough technologies. 
Sincerely,

Bradley P. Knight, MD, Northwestern University, Chicago, IL,

Thomas F. Deering, MD, Piedmont Heart Institute, Atlanta GA,

Michael R. Gold, Medical University of South Carolina, Charleston, SC,

Suneet Mittal, MD, FACC, FHRS, Valley Health, NY, NY,

Jagmeet P. Singh, MD, DPhil FACC, FHRS, Harvard University, Boston, MA

\section{References .}

1. Knight BP. Delay in Insurance Preauthorization for a Defibrillator Nearly Costs Patient with Hypertrophic Cardiomyopathy Her Life. EP Lab Digest. July 2020.

2. Reynolds D, Duray GZ, Omar R, et al., for the Micra Transcatheter Pacing Study Group. A Leadless Intracardiac Transcatheter Pacing System. N Engl J Med 2016; 374:533-541 DOI: 10.1056/NEJMoa1511643.

3. https://www.regulations.gov/document/CMS-2020-0098-0347 (Accessed April 5, 2021).

4. Bach PB. After 4 Years of Trump, Medicare and Medicaid Badly Need Attention. New York Times. March 29, 2021. https://www.nytimes.com/2020/12/01/opinion/trump-medicare-medicaid.html.

5. Rathi VK, Johnston JL, Ross JS, Dhruba SS. Medicare's New Device-Coverage Pathway - Breakthrough or Breakdown? N Engl J Med March 25, 2021. 384;12. 